\title{
An Optimized Grey Dynamic Model for Forecasting the Output of High-Tech Industry in China
}

\author{
Zheng-Xin Wang ${ }^{1}$ and Ling-Ling Pei ${ }^{2}$ \\ ${ }^{1}$ School of Economics \& International Trade, Zhejiang University of Finance \& Economics, Hangzhou 310018, China \\ ${ }^{2}$ School of Business Administration, Zhejiang University of Finance \& Economics, Hangzhou 310018, China \\ Correspondence should be addressed to Zheng-Xin Wang; jenkin107@hotmail.com
}

Received 17 September 2013; Revised 12 January 2014; Accepted 19 January 2014; Published 24 February 2014

Academic Editor: Jitao Sun

Copyright (c) 2014 Z.-X. Wang and L.-L. Pei. This is an open access article distributed under the Creative Commons Attribution License, which permits unrestricted use, distribution, and reproduction in any medium, provided the original work is properly cited.

The grey dynamic model by convolution integral with the first-order derivative of the 1-AGO data and $n$ series related, abbreviated as GDMC $(1, n)$, performs well in modelling and forecasting of a grey system. To improve the modelling accuracy of GDMC(1, $n), n$ interpolation coefficients (taken as unknown parameters) are introduced into the background values of the $n$ variables. The parameters optimization is formulated as a combinatorial optimization problem and is solved collectively using the particle swarm optimization algorithm. The optimized result has been verified by a case study of the economic output of high-tech industry in China. Comparisons of the obtained modelling results from the optimized GDMC $(1, n)$ model with the traditional one demonstrate that the optimal algorithm is a good alternative for parameters optimization of the $\operatorname{GDMC}(1, n)$ model. The modelling results can assist the government in developing future policies regarding high-tech industry management.

\section{Introduction}

Grey system theory $[1,2]$, a rising interdiscipline in uncertainty, takes the uncertain systems of "small samples and incomplete information" with "partial known information and partial unknown information" as the research object. This theory extracts valuable information mainly by generating and developing the "partial" known information. Then, the accurate demonstration of the system running behavior and the evolvement rule is done and effective monitoring is achieved. Grey system theory can be used like this to deal with the practical problems in a situation where the sample data size is not large enough to demonstrate the statistical law or the deterministic law of the system behavior.

At present, the most widely used grey model $\mathrm{GM}(1,1)$ $[1,2]$ - a first-order one-variable grey differential equationis proposed based on the aforementioned principle. Its modelling principle does not depend on distribution information from the raw data but on the application of a first-order accumulative generation operation (1-AGO), to make the sequence display grey exponent law behavior as a whole. Based on this, a first-order grey differential equation is constructed and solved. The forecast values are then derived from the first-order inverse accumulative generating operation (1IAGO). Due to the fact that the construction of $\operatorname{GM}(1,1)$ does not require a large sample and it is easy to build and calculate, $\operatorname{GM}(1,1)$ and its improved variant models have been widely used [3-6].

The $\operatorname{GM}(1, n)$ model [1], with $n-1$ relative variables acting as an associated series besides the predicted series, is a basic grey multivariable model. The forecasts of a time series $X_{1}^{(0)}$ may be considerably improved by using information coming from some associated series $X_{i}^{(0)}, i=2,3, \ldots, n$. This is particularly true if changes in $X_{1}^{(0)}$ tend to be anticipated by changes in $X_{i}^{(0)}, i=2,3, \ldots, n$. Nevertheless, the solution of the whitening differential equation for $\operatorname{GM}(1, n)$ is rough [7]-it can easily result in large errors in actual forecasting applications. Thus, for a long time, this model has been little used. In fact, the only existing research applications have proceeded on the basis of improved models [8-10]. The grey model with convolution integral, $\operatorname{GMC}(1, n)$, has been proposed by Tien [11]. This is a new model which seeks to improve the traditional $\operatorname{GM}(1, n)$ model. Theoretically, the modelling values produced by $\operatorname{GMC}(1, n)$ are the exact solution of the traditional $\operatorname{GM}(1, n)$ model and the grey control parameter 
(similar to that of the $\operatorname{GM}(1,1)$ model) introduced into the model. When the number of variables in the model $n=1$, the $\operatorname{GMC}(1, n)$ model reduces to $\operatorname{GM}(1,1)$. Using $\operatorname{GMC}(1, n)$ greatly improves the forecasting accuracy of the multivariable grey model and has been successfully applied to various different fields [11-13]. The multivariable grey model $\operatorname{GDMC}(1, n)[14]$ is based on the model $\operatorname{GMC}(1, n)$. The higher frequency components of the 1-AGO data of the associated series were significant for the grey prediction system and the first-order derivatives of 1-AGO data of the associated series were added into the $\operatorname{GDMC}(1, n)$ model.

In this study, $n$ interpolation coefficients are introduced into the background values of the variables in $\operatorname{GDMC}(1, n)$ model. Then, by aiming to minimize the modelling error, the optimal parameters are solved using the particle swarm optimization algorithm. Thereby, the adaptability and forecasting accuracy of $\operatorname{GDMC}(1, n)$ to real data are enhanced. The remainder of this paper is organized as follows. Section 2 explains the modelling methods, including the traditional $\operatorname{GDMC}(1, n)$ and the optimization method. In Section 3, the traditional $\operatorname{GDMC}(1, n)$ and the optimized $\operatorname{GDMC}(1, n)$ are applied to forecast the output of high-tech industry in China. Finally, conclusions are presented in Section 4.

\section{Methodology}

2.1. The Representation of the Grey Dynamic Model with Convolution Integral. Suppose that pairs of observations $X^{(0)}=$ $\left(X_{1}^{(0)}, X_{2}^{(0)}, \ldots, X_{n}^{(0)}\right)$ are available at equispaced time intervals consisting of $n-1$ inputs $X_{2}^{(0)}, X_{3}^{(0)}, \ldots, X_{n}^{(0)}$ and an output $X_{1}^{(0)}$ from some dynamic system. The existing $\operatorname{GDMC}(1, n)$ modelling process [14] is carried out as follows. Consider the original predicted series

$$
X_{1}^{(0)}=\left\{X_{1}^{(0)}(r p+1), X_{1}^{(0)}(r p+2), \ldots, X_{1}^{(0)}(r p+r)\right\}
$$

and the original associated series

$$
X_{i}^{(0)}=\left\{X_{i}^{(0)}(1), X_{i}^{(0)}(2), \ldots, X_{i}^{(0)}(r)\right\}, \quad i=2,3, \ldots, n .
$$

Then the 1-AGO data for $X_{1}^{(0)}, X_{2}^{(0)}, \ldots, X_{n}^{(0)}$ are given by the following equations, respectively:

$$
\begin{gathered}
X_{1}^{(1)}(r p+t)=\sum_{j=1}^{t} X_{1}^{(0)}(r p+j), \quad t=1,2, \ldots, r, \\
X_{i}^{(1)}(t)=\sum_{j=1}^{t} X_{i}^{(0)}(j), \quad t=1,2, \ldots, r, j=2,3, \ldots, n .
\end{gathered}
$$
series

The grey forecasting model based on the predicted 1-AGO

$$
X_{1}^{(1)}=\left\{X_{1}^{(1)}(r p+1), X_{1}^{(1)}(r p+2), \ldots, X_{1}^{(1)}(r p+r)\right\}
$$

and the associated 1-AGO series

$$
X_{i}^{(1)}=\left\{X_{i}^{(1)}(1), X_{i}^{(1)}(2), \ldots, X_{i}^{(1)}(r)\right\}, \quad i=2,3, \ldots, n,
$$

is given by the following differential equation:

$$
\begin{aligned}
& \frac{d X_{1}^{(1)}(r p+t)}{d t}+b_{1} X_{1}^{(1)}(r p+t) \\
& =b_{2}+\sum_{i=2}^{n}\left[b_{2 i-1} \frac{d X_{i}^{(1)}(t)}{d t}+b_{2 i} X_{i}^{(1)}(t)\right],
\end{aligned}
$$

where $b_{1}$ and $b_{2}$ are the developmental coefficient and the grey control parameter, respectively, and $\left(b_{2 i-1}, b_{2 i}\right)$ are the associated coefficients corresponding to the associated series $X_{i}^{(0)}, i=2,3, \ldots, n$, respectively, $r$ is the number of entries for model building, $r p$ is a period of delay, and $b_{1}, b_{2}, \ldots, b_{2 n}$ are model parameters to be estimated.

Equation (7) is called the grey dynamic model by convolution integral with the first-order derivative of the 1-AGO data and $n$ series related, abbreviated as $\operatorname{GDMC}(1, n)$ - the "1" represents the first-order derivative of the 1-AGO series of $X_{1}^{(1)}$ and the " $n$ " represents the total of $n$ relative series introduced into the grey differential equation.

2.2. The Evaluation of Parameters $b_{1}, b_{2}, \ldots$, and $b_{2 n}$. The grey derivative for the first-order grey differential equation with 1 -AGO is conventionally represented as

$$
\frac{d X_{1}^{(1)}(r p+t)}{d t}=\lim _{\Delta t \rightarrow 0} \frac{X_{1}^{(1)}(r p+t)-X_{1}^{(1)}(r p+t-\Delta t)}{\Delta t} .
$$

When $\Delta t \rightarrow 1$,

$$
\begin{aligned}
\frac{d X_{1}^{(1)}(r p+t)}{d t} & =\frac{\Delta X_{1}^{(1)}(r p+t)}{\Delta t} \\
& =X_{1}^{(1)}(r p+t)-X_{1}^{(1)}(r p+t-1) \\
& =X_{1}^{(0)}(r p+t) .
\end{aligned}
$$

The background value of the grey derivative $d X_{1}^{(1)}(r p+$ $t) / d t$ is taken as the mean of $X_{1}^{(1)}(r p+t)$ and $X_{1}^{(1)}(r p+t-1)$. Those of the associated series $X_{i}^{(1)}(t)$ are also taken as the mean of $X_{i}^{(1)}(t)$ and $X_{i}^{(1)}(t-1)$ for $i=2,3, \ldots, n$, respectively, for the determination of the model parameters in $\operatorname{GDMC}(1, n)$.

The least-squares solution for the model parameters in the $\operatorname{GMC}(1, n)$ in $(7)$ for $t$ from 1 to $r$ is given by

$$
\left(b_{1}, b_{2}, \ldots, b_{2 n}\right)^{T}=\left(B^{T} B\right)^{-1} B^{T} Y_{N},
$$

where 


$$
\begin{aligned}
& B=\left[\begin{array}{ccccccccc}
-W_{1}(r p+1) & 1 & X_{2}^{(0)}(1) & W_{2}(1) & X_{3}^{(0)}(1) & W_{2}(1) & \cdots & X_{n}^{(0)}(1) & W_{n}(1) \\
-W_{1}(r p+2) & 1 & X_{2}^{(0)}(2) & W_{2}(2) & X_{3}^{(0)}(2) & W_{3}(2) & \cdots & X_{n}^{(0)}(2) & W_{n}(2) \\
\vdots & \vdots & \vdots & \vdots & \vdots & \vdots & \cdots & \vdots & \vdots \\
-W_{1}(r p+r) & 1 & X_{2}^{(0)}(r) & W_{2}(r) & X_{3}^{(0)}(r) & W_{3}(r) & \cdots & X_{n}^{(0)}(r) & W_{n}(r)
\end{array}\right], \\
& W_{i}(t)=\frac{1}{2}\left(X_{i}^{(1)}(t)+X_{i}^{(1)}(t-1)\right) \text { for } i=1,2, \ldots, n, \\
& Y_{N}=\left(X_{1}^{(0)}(r p+2), X_{1}^{(0)}(r p+3), \ldots, X_{1}^{(0)}(r p+r)\right)^{T} \text {. }
\end{aligned}
$$

2.3. The Evaluation of $\widehat{X}_{1}^{(0)}$. In summary, in the right-hand side of (7), the discrete function $f(t)$ can be obtained as

$$
\begin{array}{r}
f(t)=b_{2}+\sum_{i=2}^{n}\left[b_{2 i-1} \frac{d X_{i}^{(1)}(t)}{d t}+b_{2 i} X_{i}^{(1)}(t)\right] \\
t=0,1, \ldots, r+r f .
\end{array}
$$

The 1-AGO modelling values of the predicted series can be derived using the initial condition $\widehat{X}_{1}^{(1)}(r p+1)=X_{1}^{(1)}(r p+1)$ as

$$
\begin{aligned}
& \widehat{X}_{1}^{(1)}(r p+t) \\
& \quad=X_{1}^{(1)}(r p+1) e^{-b_{1}(t-1)}+\int_{1}^{t} e^{-b_{1}(t-\tau)} f(\tau) d \tau .
\end{aligned}
$$

The second term of the right-hand side in (13) can be evaluated approximately by the two-point Gauss numerical integration [14] with the linear assumption on $f(t)$ between any two neighboring times; we have

$$
\begin{aligned}
\widehat{X}_{1}^{(1)}(r p+1) & =X_{1}^{(1)}(r p+1)=X_{1}^{(0)}(r p+1), \\
\widehat{X}_{1}^{(1)}(r p+t) & X_{1}^{(0)}(r p+1) e^{-b_{1}(t-1)}+u(t-2) \\
\times \sum_{\tau=2}^{t}\left\{\frac{1}{2} \omega_{1} e^{-b_{1}\left(t+0.5-\tau+0.5 \lambda_{1}\right)}\right. & \\
\times & 0.5(f(\tau)+f(\tau-1)) \\
& \left.+0.5 \lambda_{1}(f(\tau)-f(\tau-1))\right], \\
+ & \frac{1}{2} \omega_{2} e^{-b_{1}\left(t+0.5-\tau+0.5 \lambda_{2}\right)} \\
\times & {[0.5(f(\tau)+f(\tau-1))} \\
& \left.\left.+0.5 \lambda_{2}(f(\tau)-f(\tau-1))\right]\right\}, \\
& t=2,3, \ldots, r+r f,
\end{aligned}
$$

where the coefficients $\omega_{1}$ and $\omega_{2}$ are both equal to 1 , the nodes $\lambda_{1}$ and $\lambda_{2}$ are equal to $-1 / \sqrt{3}$ and $1 / \sqrt{3}$, respectively, and $u(t-2)$ is the unit step function. Applying 1-IAGO to (15) yields the following modelled values together with the forecasts:

$$
\begin{array}{r}
\widehat{X}_{1}^{(1)}(r p+1)=X_{1}^{(1)}(r p+1)=X_{1}^{(0)}(r p+1), \\
\widehat{X}_{1}^{(0)}(r p+t)=\widehat{X}_{1}^{(1)}(r p+t)-\widehat{X}_{1}^{(1)}(r p+t-1), \\
t=2,3, \ldots, r+r f,
\end{array}
$$

where $r f$ is the number of entries to be forecasted or indirectly measured.

Assuming the system parameters in (7) to be constants in the postsampling period, then, by using the postsampling data combined with the data given for the corresponding associated series as a new input series, the corresponding forecasts or values of indirect measurement for the predicted series can be derived.

It is obvious that, when the number of associated series is zero, that is, when $n=1$, (7) reduces to the grey single variable forecasting model $\mathrm{GM}(1,1)$.

2.4. Evaluation of the Modelling and Forecasting Accuracy. To evaluate the accuracy of the grey models Tien [14], who proposed the $\operatorname{GDMC}(1, n)$ model, applied the root mean squared percentage error (RMSPE) to the priori sample period (RMSPEPR) and postsample period (RMSPEPO), respectively. Generally, the RMSPEPR and RMSPEPO are defined, respectively, as

$$
\begin{aligned}
\text { RMSPEPR } & =\sqrt{\frac{1}{r} \sum_{t=r p+1}^{r p+r} \frac{\left[\widehat{X}_{1}^{(0)}(t)-X_{1}^{(0)}(t)\right]^{2}}{\left[X_{1}^{(0)}(t)\right]^{2}}} \times 100 \%, \\
\mathrm{RMSPEPO} & =\sqrt{\frac{1}{r f} \sum_{t=r p+r+1}^{r p+r+r f} \frac{\left[\widehat{X}_{1}^{(0)}(t)-X_{1}^{(0)}(t)\right]^{2}}{\left[X_{1}^{(0)}(t)\right]^{2}}} \times 100 \% .
\end{aligned}
$$

2.5. Optimization of the GDMC $(1, n)$ Model Based on PSO Algorithm. In this study, to enhance the modelling and forecasting accuracy of the $\operatorname{GDMC}(1, n)$ model, $n$ interpolation coefficients $\rho_{i} \in[0,1](i=1,2, \ldots, n)$ are introduced into the background values of each of the variables 
in $\operatorname{GDMC}(1, n)$. Then the optimal $\rho_{i}, i=1,2, \ldots, n$, are calculated with the objective of minimizing RMSPEPR.

Based on the above method, the background value of the grey derivative $d X_{1}^{(1)}(r p+t) / d t$ is taken as the weighted mean of $X_{1}^{(1)}(r p+t)$ and $X_{1}^{(1)}(r p+t-1)$; namely, $W_{1}(t)=\rho_{1} X_{1}^{(1)}(r p+$ $t)+\left(1-\rho_{1}\right) X_{1}^{(1)}(r p+t-1)$. Those of the associated series $X_{i}^{(1)}(t)$ are also taken as the weighted means of $X_{i}^{(1)}(t)$ and $X_{i}^{(1)}(t-1)$; that is, $W_{i}(t)=\rho_{i} X_{i}^{(1)}(t)+\left(1-\rho_{i}\right) X_{i}^{(1)}(t-1)$ for $i=2,3, \ldots, n$ in the determination of the model parameters in $\operatorname{GDMC}(1, n)$. When $\rho_{1}=\rho_{2}=\cdots=\rho_{n}=1 / 2$, the optimized $\operatorname{GDMC}(1, n)$ model is reduced to a traditional GDMC $(1, n)$ model.

The particle swarm optimization (PSO) algorithm [15] is a population-based heuristic algorithm that simulates the social behavior as birds flocking to a promising position to achieve precise objectives in a multidimensional space. Each particle is a potential solution to the optimization problem. A particle represents a point in an $n$-dimensional space, and its status is characterized through its position and velocity. The position for the particle $i$ can be represented as $X_{i}=$ $\left(X_{i 1}, X_{i 2}, \ldots, X_{i n}\right)$. The velocity of this particle can be represented by another $n$-dimensional vector $v_{i}=\left(v_{i 1}, v_{i 2}, \ldots, v_{i n}\right)$. The best previous position of the $i$ th particle can be represented as $P_{i}=\left(P_{i 1}, P_{i 2}, \ldots, P_{i n}\right)$. The best position in the entire swarm is denoted as $P_{g}=\left(P_{g 1}, P_{g 2}, \ldots, P_{g n}\right)$. To search for the optimal solution, each particle changes its velocity and position according to the following two formulas:

$$
\begin{gathered}
v_{i j}(t+1)=\omega v_{i j}(t)+c_{1} \operatorname{rand}(\cdot)\left[P_{i j}(t)-X_{i j}(t)\right] \\
+c_{2} \operatorname{rand}(\cdot)\left[P_{g j}(t)-X_{i j}(t)\right], \\
X_{i j}(t+1)=X_{i j}(t)+v_{i j}(t+1),
\end{gathered}
$$

where $t$ means the number of iterations. $\omega$ is called the inertia weight that controls the impact of previous velocity of particle on its current one. In a general way, we let $\omega_{\min }=0.4$, $\omega_{\max }=0.9 . c_{1}$ and $c_{2}$ are positive constant parameters called acceleration factors which control the maximum step size. Typical values for $c_{1}$ and $c_{2}$ are $c_{1}=c_{2}=2 \operatorname{rand}(\cdot)$ is a random number between zero and one.

To avoid the phenomenon of "shock" when particles near global optimal solution, we can employ a linear gradient strategy for the inertia weight:

$$
\omega=\omega_{\max }-\frac{\omega_{\max }-\omega_{\min }}{t_{\max }} \cdot t
$$

where $t_{\max }$ is the total number of iterations.

This study uses the PSO algorithm to solve the optimal background value coefficients for $\operatorname{GDMC}(1, n)$ model. The specific steps are as follows.

Step 1. Generate $m$ particles randomly in the $n$-dimensional space. The position and velocity for the particle $i$ can be represented as

$$
\begin{array}{r}
\rho_{i}=\left(\rho_{i 1}, \rho_{i 2}, \ldots, \rho_{\text {in }}\right), \quad v_{i}=\left(v_{i 1}, v_{i 2}, \ldots, v_{i n}\right), \\
i=1,2, \ldots, m .
\end{array}
$$

Step 2. Set the initial position of the particles as $\rho_{1}=(0,0, \ldots$, $0)$ and generate an initial velocity vector $v_{1}$ randomly in the interval $(0,1.1]$.

Step 3. According to (10), substitute $\rho_{i}$ into the matrix $B$ and establish the fitness function

$$
F\left(\rho_{i}\right)=\sqrt{\frac{1}{r} \sum_{t=r p+1}^{r p+r} \frac{\left[\widehat{X}_{1}^{(0)}(t)-X_{1}^{(0)}(t)\right]^{2}}{\left[X_{1}^{(0)}(t)\right]^{2}}} .
$$

Step 4. Calculate the fitness value for each particle.

Step 5. Compare each particle's fitness to the global best position $p_{\text {best }}$ to adapt to the value; if it is the optimum, it can be taken as the best position; if not, turn to Step 6 .

Step 6. Update particles velocity and position according to the evolution equation according to (19).

Step 7. If stopping criteria are met, show the output and its fitness, which corresponds to the optimal parameters and the RMSPEPR, or else go back to Step 3.

\section{Forecasting the Output of High-Tech Industry in China}

Forecasting the output of high-tech industry is essential for the development of projects and policy making. As Chinese high-tech industry is neonatal and so the data relating to existing economic indices are limited, it is difficult to apply existing statistical methods of analysis and forecasting to them. As a result, little research has been conducted on quantitative forecasting of Chinese high-tech industry. In this section, the advantage of the optimized $\operatorname{GDMC}(1, n)$ model (abbreviated as $\operatorname{OGDMC}(1, n)$ ) over the traditional one is demonstrated by a real case study of high-tech industry in China.

3.1. Variables and Data. In economics theory, human resources and capital investment are the crucial factors of the economic system output [16]. The economic output of hightech industry is taken as the predicted series and is denoted as $X_{1}$. And the annual average employment and investment are adopted as the relative variables of the output of hightech industry and are denoted as $X_{2}$ and $X_{3}$, respectively. The original data relating to the output, average employment, and investment of the high-tech industry in China for 20032010 are shown in Table 1. These data are all collected from the China Statistics Yearbook on High Technology Industry (2004-2011).

3.2. Modelling $\operatorname{GDMC}(1,3)$ and $\operatorname{OGDMC}(1,3)$. In the following, the industrial output value $X_{1}$ is used as the forecasting variable. At the same time, with the average employment $X_{2}$ and the investment $X_{3}$ as relative variables, multivariable forecasting models $\operatorname{GDMC}(1,3)$ and $\operatorname{OGDMC}(1,3)$ models are established. The data of $X_{1}, X_{2}$, and $X_{3}$ from 2003 to 2010 
TABLE 1: Industrial output value, average employment, and investment of high-tech industry in China.

\begin{tabular}{lccc}
\hline $\begin{array}{l}\text { Time } \\
\text { (Year) }\end{array}$ & $\begin{array}{c}\text { Industrial output value } \\
\text { (RMB 100 million } \\
\text { yuan) }\end{array}$ & $\begin{array}{c}\text { Average } \\
\text { employment } \\
\text { (10000 persons) }\end{array}$ & $\begin{array}{c}\text { Investment } \\
\text { (RMB 100 } \\
\text { million yuan) }\end{array}$ \\
\hline $3(2003)$ & 20556 & 477 & 1423 \\
$4(2004)$ & 27769 & 587 & 1790 \\
$5(2005)$ & 34367 & 663 & 2144 \\
$6(2006)$ & 41996 & 744 & 2761 \\
$7(2007)$ & 50461 & 843 & 3388 \\
$8(2008)$ & 57087 & 945 & 4169 \\
$9(2009)$ & 60430 & 958 & 4882 \\
$10(2010)$ & 74709 & 1092 & 6945 \\
\hline
\end{tabular}

TABLE 2: The values of parameters $n, r$, and $r p$ in (7), the estimates of model parameters $b_{1}, b_{2}$, and $\left(b_{2 i-1}, b_{2 i}\right)$ in (10), and the value of RMSPEPR in (17).

\begin{tabular}{lccc}
\hline Parameter & Value & Parameter & Value \\
\hline$n$ & 3 & $b_{3}$ & 23.28 \\
$r$ & 8 & $b_{4}$ & 17.78 \\
$r p$ & 0 & $b_{5}$ & 9.30 \\
$b_{1}$ & 0.10822 & $b_{6}$ & -3.20 \\
$b_{2}$ & -5083.77 & RMSPEPR $(\%)$ & 7.07 \\
\hline
\end{tabular}

TABLE 3: The discrete function $f(t)$ for $\operatorname{GDMC}(1,3)$ in $(12)$.

\begin{tabular}{cccc}
\hline$t$ & $f(t)$ & $t$ & $f(t)$ \\
\hline 1 & 23173.06 & 5 & 68109.22 \\
2 & 33851.44 & 6 & 81192.52 \\
3 & 43829.28 & 7 & 89520.70 \\
4 & 55835.86 & 8 & 108987.60 \\
\hline
\end{tabular}

is employed as the original modelling series to construct $\operatorname{GDMC}(1,3)$ and $\operatorname{OGDMC}(1,3)$ models.

3.2.1. $\operatorname{GDMC}(1,3)$. Applying the $\operatorname{GDMC}(1,3)$ model given by (7)-(16), the values of the parameters $n, r$, and $r p$ in (7) and the estimates of the model parameters $b_{1}, b_{2}$, and $\left(b_{2 i-1}, b_{2 i}\right)$ in (10) can be obtained (Table 2). The resulting $\operatorname{GDMC}(1,2)$ model from (7) has the form

$$
\begin{aligned}
& \frac{d X_{1}^{(1)}(t)}{d t}+0.10822 X_{1}^{(1)}(t) \\
& =-5083.77+23.28 \frac{d X_{2}^{(1)}(t)}{d t}+17.78 X_{2}^{(1)}(t) \\
& +9.30 \frac{d X_{3}^{(1)}(t)}{d t}-3.20 X_{3}^{(1)}(t) \\
& t=1,2, \ldots, 8 .
\end{aligned}
$$

To sum up the right-hand side of (7), the discrete function $f(t)$ in $(12)$ for the $\operatorname{GMC}(1,2)$ model is obtained and tabulated (Table 3 ). The values of RMSPEPR in (17), respectively, are also listed in Table 2.
TABLE 4: The values of parameters $n, r, r f$, and $r p$ in (7), the estimates of model parameters $b_{1}, b_{2}$, and $\left(b_{2 i-1}, b_{2 i}\right)$ in (10), the optimized parameters $\rho_{1}, \rho_{2}$, and $\rho_{3}$, and the values of RMSPEPR in (17).

\begin{tabular}{lccc}
\hline Parameter & Value & Parameter & Value \\
\hline$n$ & 3 & $b_{5}$ & 8.23 \\
$r$ & 8 & $b_{6}$ & -1.68 \\
$r p$ & 0 & $\rho_{1}$ & 0.17624 \\
$b_{1}$ & -0.34721 & $\rho_{2}$ & 0.14669 \\
$b_{2}$ & -0.45756 & $\rho_{3}$ & 1 \\
$b_{3}$ & 34.90 & RMSPEPR $(\%)$ & 1.86 \\
$b_{4}$ & 18.76 & & \\
\hline
\end{tabular}

TABLE 5: The discrete function $f(t)$ for $\operatorname{OGDMC}(1,3)$ in $(12)$.

\begin{tabular}{cccc}
\hline$t$ & $f(t)$ & $t$ & $f(t)$ \\
\hline 1 & 27896.61 & 5 & 93126.25 \\
2 & 42762.85 & 6 & 113838.20 \\
3 & 57161.09 & 7 & 129932.20 \\
4 & 74385.06 & 8 & 160408.60 \\
\hline
\end{tabular}

TABLE 6: Levels of accuracy and their critical values.

\begin{tabular}{lcccc}
\hline Level & MRE & ADGI & RSD & PSE \\
\hline 1 & 0.01 & 0.90 & 0.35 & 0.95 \\
2 & 0.05 & 0.80 & 0.50 & 0.80 \\
3 & 0.10 & 0.70 & 0.65 & 0.70 \\
4 & 0.20 & 0.60 & 0.80 & 0.60 \\
\hline
\end{tabular}

3.2.2. $\operatorname{OGDMC}(1,3)$. Applying the $\operatorname{OGDMC}(1,3)$ model given by (7)-(16) and the optimized method, the values of the parameters $n, r$, and $r p$ in (7), the estimates of the model parameters $b_{1}, b_{2}$, and $\left(b_{2 i-1}, b_{2 i}\right)$ in (10), and the optimized parameters $\rho_{1}, \rho_{2}$, and $\rho_{3}$ can be obtained (listed in Table 4). The resulting $\operatorname{OGDMC}(1,3)$ model from $(7)$ has the form

$$
\begin{aligned}
& \frac{d X_{1}^{(1)}(t)}{d t}+0.22310 X_{1}^{(1)}(t) \\
&=-7021.86+34.90 \frac{d X_{2}^{(1)}(t)}{d t}+18.76 X_{2}^{(1)}(t) \\
&+8.23 \frac{d X_{3}^{(1)}(t)}{d t}-1.68 X_{3}^{(1)}(t), \\
& t=1,2, \ldots, 8 .
\end{aligned}
$$

To sum up the right-hand side of (7), the discrete function $f(t)$ in $(12)$ for the $\operatorname{OGDMC}(1,2)$ model is obtained and tabulated (Table 5). The values of RMSPEPR in (17) are also listed in Table 4.

3.3. Evaluation of the Grey Forecasting Models. In this study, the most commonly used four measurements in grey theory, including mean relative error (MRE), absolute degree of grey incidence (ADGI), ratio of standard deviation (RSD), and probability of small error (PSE) [2,17], are used to evaluate the accuracy of the models involved. The levels of accuracy and their critical values are given in Table 6 [17]. When all 
TABLE 7: The modelling results obtained using the $\operatorname{GDMC}(1,3)$ and $\operatorname{OGDMC}(1,3)$ models.

\begin{tabular}{|c|c|c|c|c|c|}
\hline \multirow{2}{*}{ Time (Year) } & \multirow{2}{*}{ Actual value } & \multicolumn{2}{|c|}{$\operatorname{GDMC}(1,3)$} & \multicolumn{2}{|c|}{$\operatorname{OGDMC}(1,3)$} \\
\hline & & Modelling value & Relative error & Modelling value & Relative error \\
\hline $1(2003)$ & 20556 & 20556 & 0 & 20556 & 0 \\
\hline $2(2004)$ & 27769 & 24823.94 & 0.0609 & 27308.16 & 0.0095 \\
\hline $3(2005)$ & 34367 & 32072.61 & 0.0277 & 34970.11 & 0.0073 \\
\hline $4(2006)$ & 41996 & 39183.89 & 0.0226 & 42101.73 & 0.0008 \\
\hline $5(2007)$ & 50461 & 46668.65 & 0.0217 & 49775.32 & 0.0039 \\
\hline $6(2008)$ & 57087 & 53891.31 & 0.0138 & 57470.03 & 0.0016 \\
\hline 7 (2009) & 60430 & 58551.09 & 0.0064 & 62549.36 & 0.0072 \\
\hline $8(2010)$ & 74709 & 67230.30 & 0.0204 & 72731.92 & 0.0054 \\
\hline MRE & & \multicolumn{2}{|c|}{0.0217} & \multicolumn{2}{|c|}{0.0045} \\
\hline ADGI & & \multicolumn{2}{|c|}{0.94} & \multicolumn{2}{|c|}{1.00} \\
\hline RSD & & \multicolumn{2}{|c|}{0.12} & \multicolumn{2}{|c|}{0.06} \\
\hline PSE & & \multicolumn{2}{|c|}{1.00} & \multicolumn{2}{|c|}{1.00} \\
\hline
\end{tabular}

the measured values in a forecasting model meet the requirements of the critical values listed in the table, the model is applicable to the prediction. If they fall within the ranges of levels 1 and 2, this suggests that the model has high forecasting accuracy.

3.4. Empirical Results and Discussion. The modelling results for China's high-tech industry using the two grey models described above are shown in Table 7 together with the four accuracy measurements. The results show that the $\operatorname{GDMC}(1,3)$ model and the $\operatorname{OGDMC}(1,3)$ model fall within levels 2 and 1 , respectively. The $\operatorname{OGDMC}(1,3)$ model reduces the MRE of the $\operatorname{GDMC}(1,3)$ model from 0.0217 to 0.0045 , increases the ADGI of the $\operatorname{GDMC}(1,3)$ model from 0.94 to 1.00 , and also reduces the RSD of the $\operatorname{GDMC}(1,3)$ model from 0.12 to 0.06 . This indicates that the $\operatorname{OGDMC}(1, n)$ model can improve the modelling accuracy of the traditional model $\operatorname{GDMC}(1, n)$ significantly.

Figure 1 demonstrates the degree of closeness between the modelling results of the models and the real values of the output of high-tech industry in China. Clearly, the model $\operatorname{OGDMC}(1,3)$ is closer to the actual data than the traditional one. Moreover, as can be seen from a histogram of the modelling residual errors (Figure 2), the residual error from the $\operatorname{OGDMC}(1,3)$ model is notably smaller than that of the $\operatorname{GDMC}(1,3)$. The reason for this lies in the fact that, through optimization of the background value, the modelling ability of the $\operatorname{GDMC}(1,3)$ model can be further improved in the OGDMC $(1,3)$ model. The model coefficients $b_{3}, b_{4}, b_{5}$, and $b_{6}$ can reveal the role and importance of employment and investment and their impact on the output of high-tech industry.

\section{Conclusions}

This study presents a PSO-algorithm-based optimization method for the grey dynamic model by convolution integral with the first-order derivative of the 1-AGO data and $n$ series related. According to empirical modelling results of the high-tech industry in China, the modelling accuracy of the

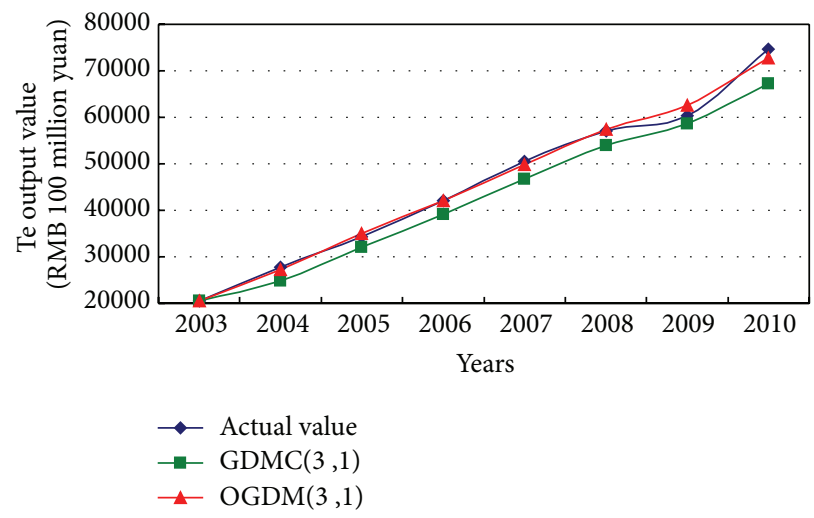

FIGURE 1: The output value and the modelling curves obtained using the $\operatorname{GDMC}(1,3)$ and $\operatorname{OGDMC}(1,3)$ models.

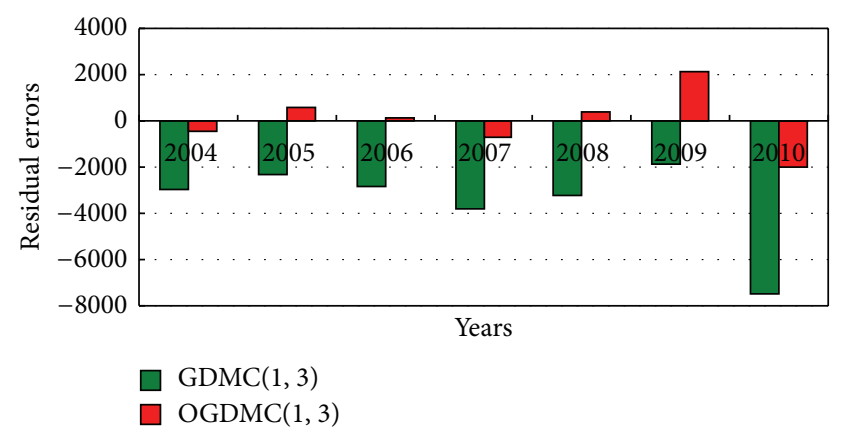

FIGURE 2: The residual errors occurring in the $\operatorname{GDMC}(1,3)$ and $\operatorname{OGDMC}(1,3)$ models.

traditional GDMC $(1, n)$ model can be effectively increased using a background value optimization method as proposed in this study. This is important in practice as the government of high-tech industry needs to make decisions based on traditional parameter solutions which may be constrained by an incorrect local optimal solution.

Due to the lack of more additional data published by China's Statistic Department at present, the out-of-sample 
and the time-delayed forecasting results of this study have not fully been validated. Therefore, in future work, the outof-sample and the time-delayed forecasting using both the $\operatorname{GDMC}(1, n)$ and the $\operatorname{OGDMC}(1, n)$ needs to be taken into account after more data is released by China's Statistic Department. In addition, the grey forecasting model established in this study merely considers two basic input factors, that is, capital and labor, while ignoring the technical level. The main reason lies in the fact that sample data are rather limited in the short development of China's high-tech industries. Moreover, it is challengeable to estimate the technology level of high-tech industries using small sample data. To address this problem, further researches are also needed.

\section{Conflict of Interests}

The authors declare that there is no conflict of interests regarding the publication of this paper.

\section{Acknowledgments}

The authors are grateful to the editors and the anonymous reviewers for their insightful comments and suggestions. The authors also thank the National Natural Science Foundation of China (Grant no. 71101132), the Philosophy and Social Science Foundation of Zhejiang Province, China (Grant no. 13ZJQN029YB), and the Academic Climbing Project for Young and Middle-Aged Leading Academic in the Universities of Zhejiang Province, China (Grant no. PD2013275), for financially supporting this study.

\section{References}

[1] J. L. Deng, The Elements on Grey Theory, Huazhong University of Science and Technology Press, Wuhan, China, 2nd edition, 2002.

[2] S. F. Liu and Y. Lin, Grey Information Theory and Practical Applications, Springer, London, UK, 1st edition, 2006.

[3] D. Li, C. Chang, C. Chen, and W. Chen, "Forecasting shortterm electricity consumption using the adaptive grey-based approach—an Asian case," Omega, vol. 40, no. 6, pp. 767-773, 2012.

[4] C.-H. Wang and L.-C. Hsu, "Using genetic algorithms grey theory to forecast high technology industrial output," Applied Mathematics and Computation, vol. 195, no. 1, pp. 256-263, 2008.

[5] I. J. Lu, C. Lewis, and S. J. Lin, "The forecast of motor vehicle, energy demand and $\mathrm{CO}_{2}$ emission from Taiwan's road transportation sector," Energy Policy, vol. 37, no. 8, pp. 2952-2961, 2009.

[6] C. J. Chang, D. C. Li, W. L. Dai, and C. C. Chen, "Utilizing an adaptive grey model for short-term time series forecasting: a case study of wafer-level packaging," Mathematical Problems in Engineering, vol. 2013, Article ID 526806, 6 pages, 2013.

[7] T.-L. Tien, "A research on the grey prediction model GM $(1, n)$," Applied Mathematics and Computation, vol. 218, no. 9, pp. 49034916, 2012.

[8] L. Hsu, "Forecasting the output of integrated circuit industry using genetic algorithm based multivariable grey optimization models," Expert Systems with Applications, vol. 36, no. 4, pp. 7898-7903, 2009.

[9] L. Hsu and C. Wang, "Forecasting integrated circuit output using multivariate grey model and grey relational analysis," Expert Systems with Applications, vol. 36, no. 2, pp. 1403-1409, 2009.

[10] Y. X. Luo, X. Wu, M. Li, and A. H. Cai, "Grey dynamic model $\operatorname{GM}(1, N)$ for the relationship of cost and variability," Kybernetes, vol. 38, no. 3-4, pp. 435-440, 2009.

[11] T. Tien, "The indirect measurement of tensile strength of material by the grey prediction model $\operatorname{GMC}(1, n)$, , Measurement Science and Technology, vol. 16, no. 6, pp. 1322-1328, 2005.

[12] W.-Y. Wu and S.-P. Chen, "A prediction method using the grey model GMC $(1, n)$ combined with the grey relational analysis a case study on internet access population forecast," Applied Mathematics and Computation, vol. 169, no. 1, pp. 198-217, 2005.

[13] C. Su, C. Chen, W. Liu, and H. Lai, "Estimation for inner surface geometry of furnace wall using inverse process combined with grey prediction model," International Journal of Heat and Mass Transfer, vol. 52, no. 15-16, pp. 3595-3605, 2009.

[14] T. Tien, "The indirect measurement of tensile strength for a higher temperature by the new model $\operatorname{IGDMC}(1, n)$," Measurement, vol. 41, no. 6, pp. 662-675, 2008.

[15] S. Gao and J. Y. Yang, Swarm Intelligence Algorithm and Its Application, China Water Power Press, Beijing, China, 2006.

[16] Z. N. Li, Econometrics, Higher Education Press, Beijing, China, 2nd edition, 2010.

[17] D. Li, C. Chang, W. Chen, and C. Chen, "An extended grey forecasting model for omnidirectional forecasting considering data gap difference," Applied Mathematical Modelling, vol. 35, no. 10, pp. 5051-5058, 2011. 


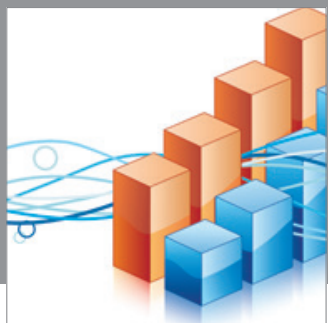

Advances in

Operations Research

mansans

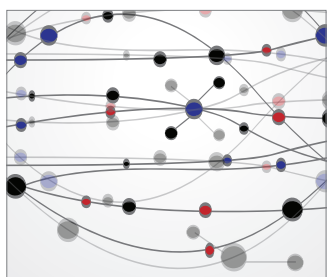

The Scientific World Journal
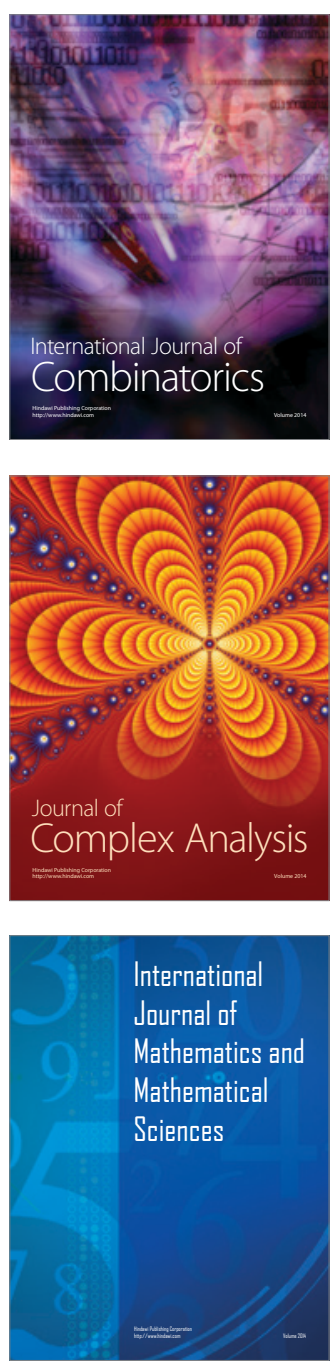
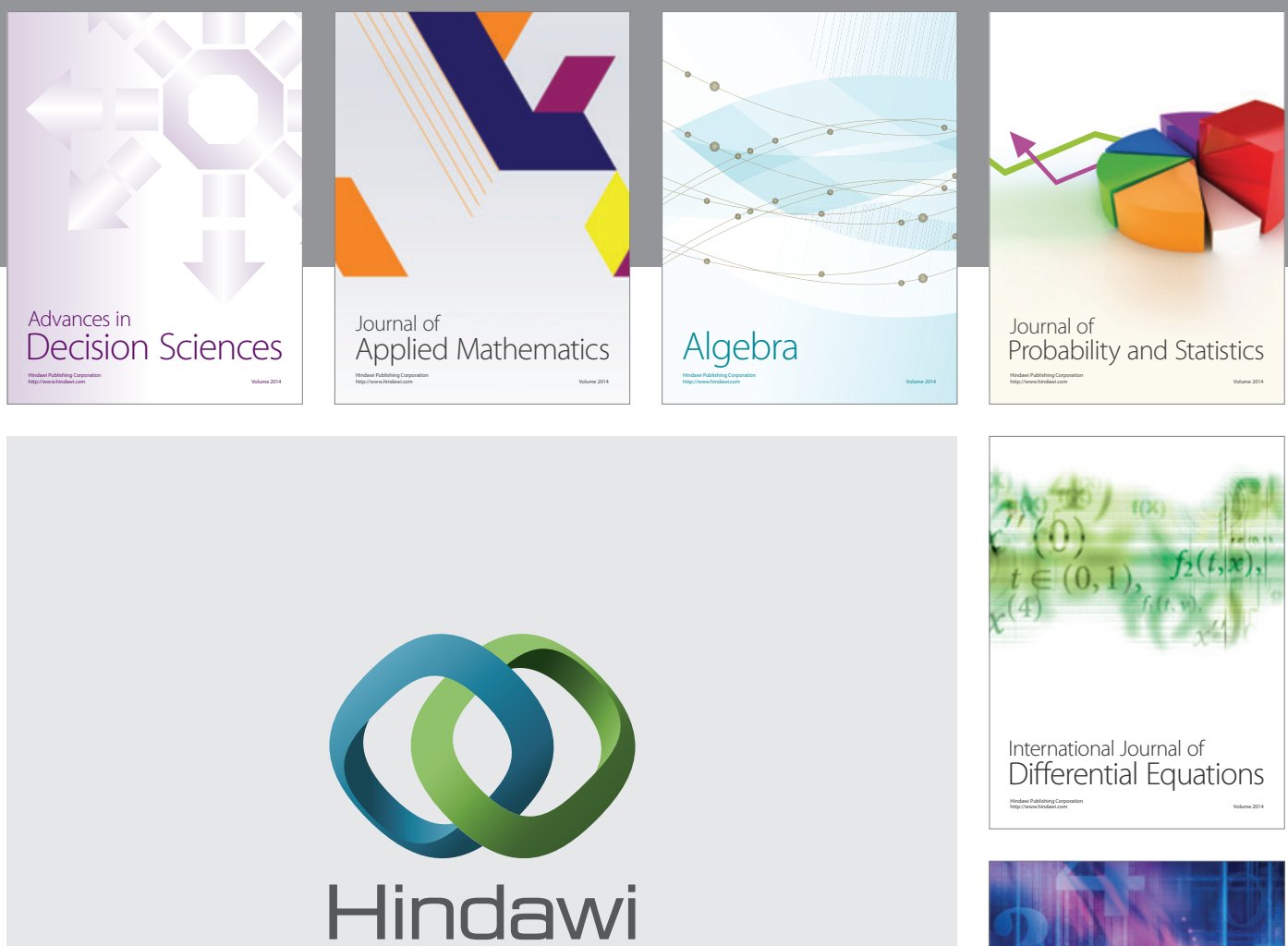

Submit your manuscripts at http://www.hindawi.com
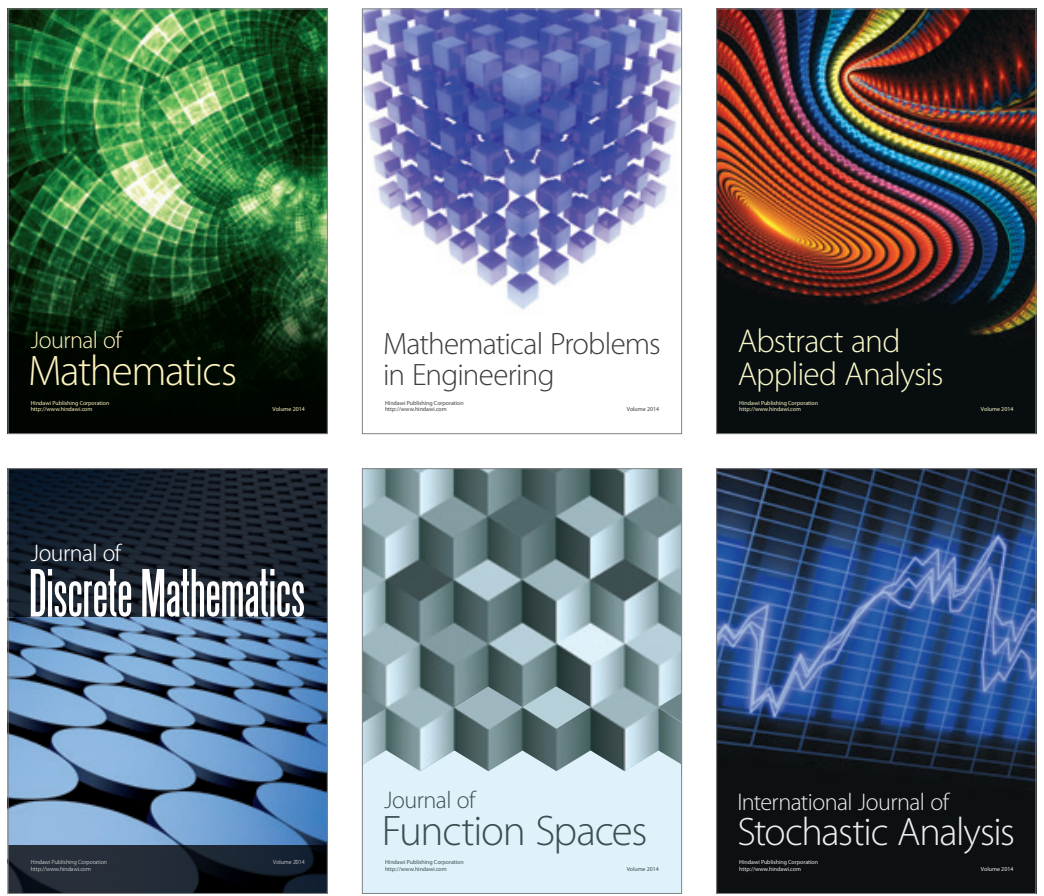

Journal of

Function Spaces

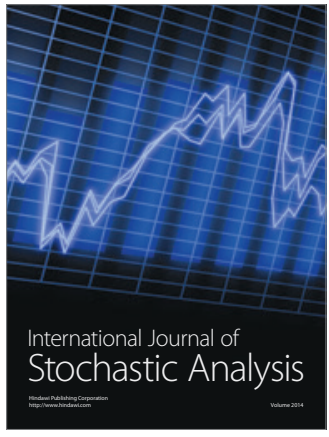

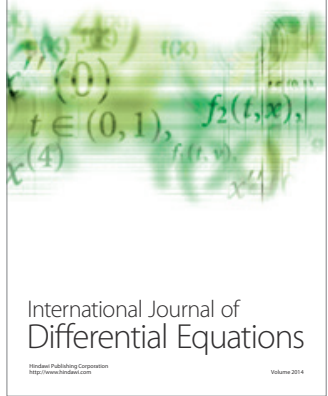
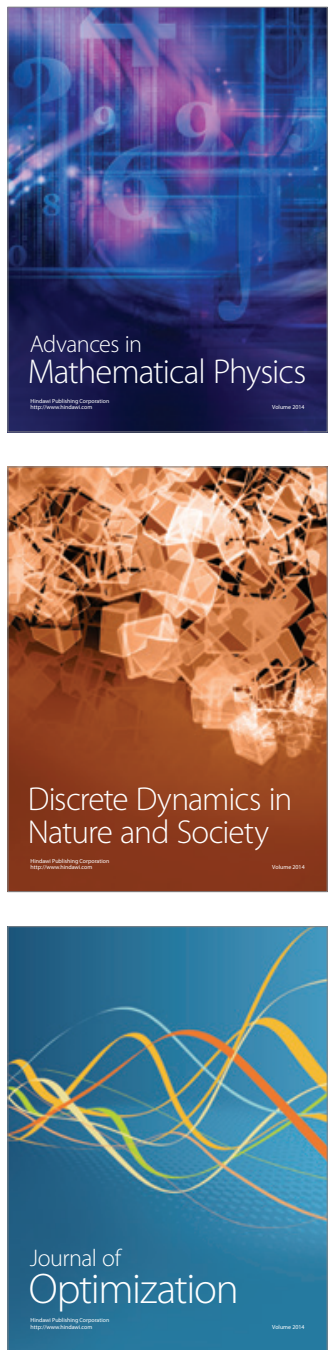